Annie Cuyt • Vigdis Brevik Petersen Brigitte Verdonk $\bullet$ Haakon Waadeland William B. Jones

\title{
Handbook of Continued Fractions for Special Functions
}

With contributions by

Franky Backeljauw • Catherine Bonan_Hamada

Verified numerical output

Stefan Becuwe • Annie Cuyt

43 Springer 


\section{TABLE OF CONTENTS}

Preface $\quad$ xi

Notation .................................................................................. xiii

$0 \quad$ General considerations ..................................................... 1

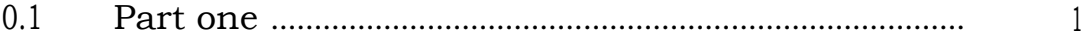

$0.2 \quad$ Part two .............................................................................. 2

$0.3 \quad$ Part three ...................................................................... 2

\section{Part I: BASIC THEORY}

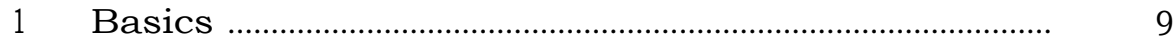

$1.1 \quad$ Symbols and notation .................................................... 9

1.2 Definitions .................................................................... 10

1.3 Recurrence relations ..................................................... 13

1.4 Equivalence transformations ......................................... 15

$1.5 \quad$ Contractions and extensions ......................................... 16

1.6 Continued fractions with prescribed approximants ..... 18

$\begin{array}{lll}1.7 & \text { Connection between continued fractions and series .... } & 19\end{array}$

1.8 Periodic and limit periodic continued fractions ............ 21

1.9 Tails of continued fractions ............................................... 23

1.10 Continued fractions over normed fields ........................ 26

1.11 Generalisations of continued fractions ........................ 28

2 Continued fraction representation of functions .......... 29

$2.1 \quad$ Symbols and notation .................................................... 29

2.2 Correspondence ................................................................... 30

2.3 Families of continued fractions ....................................... 35

2.4 Correspondence of C-fractions ....................................... 39

$2.5 \quad$ Correspondence of P-fractions ......................................... 40

2.6 Correspondence of J-fractions and T-fractions ........... 41

2.7 Correspondence and three-term recurrences ................. 42

3 Convergence criteria ................................................................. 45

3.1 Some classical theorems ................................................. 45

3.2 Convergence sets and value sets ..................................... 47

3.3 Parabola and oval theorems .......................................... 49 
3.4 Correspondence and uniform convergence ........................ 52

3.5 Periodic and limit periodic continued fractions ............... 53

3.6 Convergence and minimal solutions ..................................... 56

$4 \quad$ Pade approximants .................................................................. 59

4.1 Definition and notation ....................................................... 59

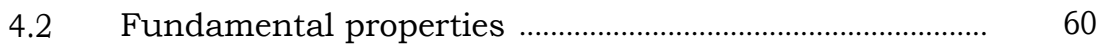

4.3 Connection with regular C-fractions ..................................... 64

4.4 Connection with P-fractions .................................................... 65

4.5 Extension of the Pa\& table ....................................................... 67

4.6 Connection with M-fractions and the M-table ............... 68

4.7 Convergence of Pa\& approximants ...................................... $\quad 70$

4.8 Formal orthogonality property ............................................. $\quad 72$

$5 \quad$ Moment theory and orthogonal functions ......................... $\quad 77$

5.1 Moment theory ....................................................................... 77

5.2 Stieltjes transforms ................................................................. 85

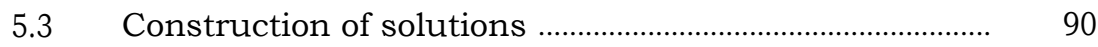

5.4 Orthogonal polynomials ........................................................ 91

5.5 Monic orthogonal polynomials an IR and J-fractions .... 92

5.6 Szegö polynomials and PPC-fractions .............................. 100

$\begin{array}{lll}5.7 & \text { Orthogonal Laurent polynomials and APT-fractions } & 102\end{array}$

\section{Part II: NUMERICS}

$6 \quad$ Continued fraction construction ................................................. 107

6.1 Regular C-fractions ............................................................. 107

6.2 C-fractions ........................................................................ 113

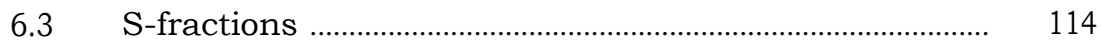

6.4 P-fractions .............................................................................. 114

$6.5 \mathrm{~J}$-fractions ..................................................................... 120

$6.6 \quad$ M-fractions ..................................................................... 122

6.7 Positive T-fractions ................................................................... 124

6.8 Thiele fractions .................................................................. 125

$7 \quad$ Truncation error bounds ................................................................. 129

7.1 Parabola theorems ............................................................... 129

7.2 The oval sequence theorem …................................................. 131

7.3 The interval sequence theorem ............................................ 136

7.4 Specific a priori bounds for S-fractions ............................. 138

7.5 A posteriori truncation error bounds ................................ 140 
7.6 Tails and truncation error bounds ......................................... 143

7.7 Choice of modification ................................................................ 143

8 Continued fraction evaluation ......................................................... 149

8.1 The effect of finite precision arithmetic ............................... 149

8.2 Evaluation of approximants .................................................. 152

8.3 The forward recurrence and minimal solutions ................. 154

8.4 Round-off error in the backward recurrence ........................ 156

\section{Part III: SPECIAL FUNCTIONS}

$9 \quad$ On tables and graphs ............................................................................. 163

$9.1 \quad$ Introduction ...................................................................... 163

9.2 Comparative tables …............................................................ 163

9.3 Reliable graphs [............................................................ 168

10 Mathematical constants .............................................................. 175

10.1 Regular continued fractions ................................................ 175

10.2 Archimedes' constant, symbol 7r ......................................... 176

10.3 Euler's number, base of the natural logarithm ................. 178

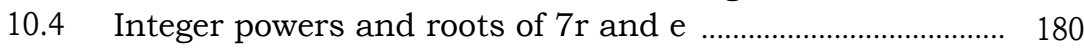

10.5 The natural logarithm, $\ln (2)$................................................. 181

10.6 Pythagoras' constant, the square root of two .................... 183

10.7 The cube root of two ............................................................... 183

10.8 Euler's constant, symbol -), ...................................................... 185

10.9 Golden ratio, symbol 0 ............................................................ 185

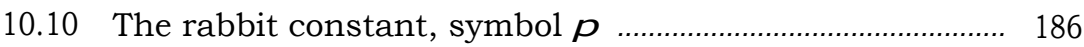

10.11 Apery's constant, ((3) ........................................................... 188

10.12 Catalan's constant, symbol $C$.................................... 189

10.13 Gompertz' constant, symbol $G$........................................ 190

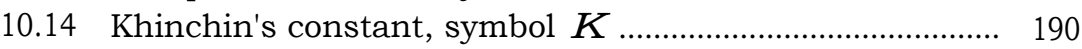

11 Elementary functions ................................................................ 193

11.1 The exponential function ...................................................... 193

11.2 The natural logarithm ........................................................ 196

11.3 Trigonometric functions ............................................................. 200

11.4 Inverse trigonometric functions .............................................. 204

11.5 Hyperbolic functions ............................................................... 210

11.6 Inverse hyperbolic functions ................................................ 213

11.7 The power function ........................................................... 217 
12 Gamma function and related functions

12.1 Gamma function .................................................................. 221

12.2 Binet function ................................................................... 224

12.3 Polygamma functions ......................................................... 229

12.4 Trigamma function ........................................................... 232

12.5 Tetragamma function .................................................... 235

12.6 Incomplete gamma functions ...................................... 238

13 Error function and related integrals ........................................... 253

13.1 Error function and Dawson's integral ............................. 253

13.2 Complementary and complex error function .................. 261

13.3 Repeated integrals ............................................................. 268

13.4 Fresnel integrals ........................................................... 269

14 Exponential integrals and related functions ......................... 275

14.1 Exponential integrals ........................................................ 275

14.2 Related functions ......................................................... 285

15 Hypergeometric functions .............................................................. 291

15.1 Definition and basic properties ....................................... 291

15.2 Stieltjes transform ........................................................ 295

15.3 Continued fraction representations ................................. 295

15.4 Pade approximants ............................................................. 309

$15.5 \quad$ Monotonicity properties ..................................................... 313

15.6 Hypergeometric series $\boldsymbol{F}_{\boldsymbol{q}} \quad 315$

16 Confluent hypergeometric functions ......................................... 319

$16.1 \quad$ Kummer functions ............................................................. 319

16.2 Confluent hypergeometric series 2Fo .......................................... 330

16.3 Confluent hypergeometric limit function .......................... 333

$16.4 \quad$ Whittaker functions ..................................................... 334

16.5 Parabolic cylinder functions ............................................ 337

17 Bessel functions ............................................................................ 343

$17.1 \quad$ Bessel functions .................................................................. 343

17.2 Modified Bessel functions ................................................ 356

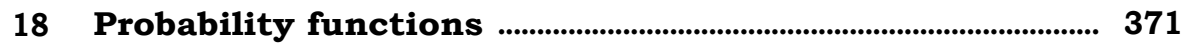

18.1 Definitions and elementary properties ........................... 371

18.2 Normal and log-normal distributions ............................... 373

18.3 Repeated integrals ......................................................... 377

18.4 Gamma and chi-square distribution .............................. 378 
18.5 Beta, F- and Student's t-distributions

19 Basic hypergeometric functions 391

19.1 Definition and basic properties 391

19.2 Continued fraction representations 395

19.3 Higher order basic hypergeometric functions 399

Bibliography 401

Index 